\title{
Surface Energy Induced Patterning of Polymer Nanostructures for Cancer Diagnosis and Therapy
}

\author{
Wenchuang (Walter) Hu, Member, IEEE, Fern Yoon, Adam Crouch, Student Member, IEEE, Li Tao, \\ Student Member, IEEE, Heather Hillebrenner, Jagadeesh Setti Guthi, Moon Kim, and Jinming Gao
}

\begin{abstract}
We have developed a new simple method to pattern discrete polymer micro and nanostructures. A Si template is patterned by lithography and selective surface treatment to have spatially different surface energies that induce microfluidic self-patterning of a spincoated polymer layer. Biocompatible diblock co-polymer and SU8 are patterned using this method to form monodisperse and shape specific microstructures. After patterning, these particles are lifted off the template surface into aqueous solution. The template is then cleaned and re-used. These freestanding polymer particles with uniform and precise spherical morphology can be used as carriers for drug and imaging agents for biomedical applications.
\end{abstract}

\section{INTRODUCTION}

$\mathrm{T}$ HROUGH the engineering of bioactive nanostructures and processes, a new wave of medical innovation may be stimulated: for example, functionalized nanoparticles or nanoscale capsules for advanced delivery, targeting, and recognition [1-6]; nano-probes or nanomaterials for early detection of disease and ultra-sensitive imaging [7][8]; nanostructured scaffolds to promote tissue regeneration [9-11]. For these exploitations of nanotechnology, the ability to fabricate structures with nanometer precision is of fundamental importance. Cost effective fabrication methods are highly desired to produce polymeric nanostructures with precise control of geometry, dimensions, and uniformity, but without changing/damaging their active bio-functionality.

Available fabrication methods are mainly divided into "bottom-up" self-assembly and "top-down" nanolithography in conjunction with pattern transfer methods such as etching and liftoff processes. However for self-assembly the control of uniformity, 3D geometry, and transfer to substrate are difficult [5]. For lithography, patterning functional organic materials is a challenge. Pattern transfer using liftoff will involve strong solvents, which is not compatible for organic materials. Pattern

The authors thank Dr. Steve Levene for the use of their AFM. This work is partially sponsored by the Moncrief Foundation.

W. Hu, M. Kim, F. Yoon, A. Crouch, and L. Tao are with Nanoscale Integration Laboratory, Department of Electrical Engineering, University of Texas at Dallas, Richardson, TX 75083, USA (e-mail: Walter.Hu@utdallas.edu)

H. Hillebrenner, J. S. Guthi, and J. M. Gao are with Simmons Comprehensive Cancer Center, University of Texas Southwestern Medical Center, TX 75390, USA (e-mail: Jinming.Gao@UTSouthwestern.edu) transfer with etching will damage or change functionality of materials as well. Nanoimprint lithography (NIL) [12] and soft lithography [13] are feasible methods to produce polymer structures by thermal or UV induced molding. Other strategies to utilize polymer capillary flow for patterning of micro and nanostructures have also been reported, such as edge lithography [14] [15] and lithography induced self-assembly [16]. However, for NIL and its derivatives, a layer of residue is commonly formed and an invasive etching step is still needed to form separated particles. Recently, DeSimone et al. extended soft lithography with perfluoro-polymer molds on non-wetting substrates to fabricate monodispersed particles with controlled morphology [17]. However in order to make discrete polymer structures, liquid precursors must be used, which limits its application to solid phase materials.

Here we present a simple process to pattern discrete polymer micro and nanostructures through discrete surface energy induced microfluidic self-patterning. This method is based on patterning a template to have spatially different surface energies via conventional lithography and selective surface treatment. Then the discrete surface energies on the substrate induce microfluidic self-patterning of materials that are deposited on the surface. The major advantage of this method is that once the template is made, monodispersed particles can be produced by simply putting the sample on a hotplate to induce polymer reflow. After the particles have been harvested, the template can be re-used for "lithography-less" fabrication of particles.

\section{EXPERIMENTS AND RESULTS}

\section{A. SEIP process}

Figure 1 illustrates the process of the surface energy induced patterning (SEIP). First, nanoimprint or photolithography is used to define openings in the resist. Then trichlorosilane self-assembled monolayers (SAMS), such as (Heptadecafluoro-1,1,2,2 - tetrahydrodecyl) trichlorosilane (FDTS) is covalently bonded to the hydroxyl groups on the surface of the openings. The resist is removed and the rest of Si is modified with methacryloxypropyl trichlorosilane (MOPTS). A template with spatially different surface energies is formed and then spincoated with polymer. Heating the deposited polymer film above its glass transition temperature (Tg) on a hot plate allows the material to re-flow and self-pattern to the 
areas of high surface energy (MOPTS treated Si), leaving empty space at the areas of low surface energy (FDTS treated Si). In this way, discrete polymer structures can be made. For freestanding particles, these structures can be harvested from the template into aqueous solution. Then the template can be cleaned and re-used to produce more.

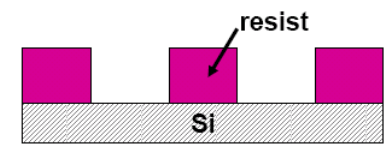

a) Pattern resist by lithography

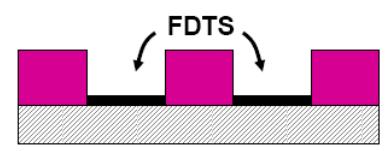

b) Treat surface with FDTS

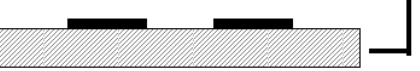

c) Remove polymer mask d) Treat surface with MOPTS

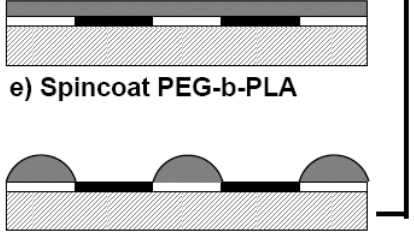

f) Heat to $100^{\circ} \mathrm{C}$ for re-flow

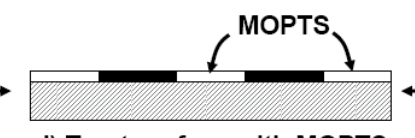

Fig. 1. Schematic of SEIP process flow

\section{B. Template fabrication}

Photolithography and NIIL were used to make the discrete surface energy templates. Templates with $0.5 \mu \mathrm{m}$ and $1 \mu \mathrm{m}$ wide chemical lines were produced using nanoimprint lithography into $500 \mathrm{~nm}$ thick $950 \mathrm{~K}$ poly (methyl methacrylate) (PMMA) on an Obducat 2.5 nanoimprinter at a temperature of $180^{\circ} \mathrm{C}$ and a pressure of $6 \mathrm{MPa}$ for $10 \mathrm{~min}$. The PMMA residue layer was then removed by reactive ion etching. Exposed $\mathrm{Si}$ areas were then soaked in the FDTS ( 0.1\% in n-heptane) solution. PMMA was found to be a good mask material to selectively treat the substrates, keeping the n-heptane solution from penetrating and treating the masked areas. The PMMA was then removed with acetone. The template was then soaked in MOPTS solution in n-heptane to coat the rest of the $\mathrm{Si}$ template. With similar process, templates with $2 \mu \mathrm{m}$ dots were made by photolithography in S1813 resist over over a 1 sq.in.Si Sample. The SAMS treatment was the same as the nanoimprint process. After the templates were made, AFM was used to evaluate their surface conditions. As shown in Fig. 2, AFM image of FDTS treated Si (before MOPTS treatment) shows that the FDTS thickness measured $0.65-0.94 \mathrm{~nm}$. After MOPTS treatment, the surface topography is very small. Topography image shows no significant patterns (Fig. 2b), while phase image shows clear dot patterns (Fig. 2c). The phase image indicates that the friction coefficients in the dots and in the spacing areas are different. Therefore the heterogeneous surface energies were formed on the Si template. Since the maximum topography difference of the template is only about $1 \mathrm{~nm}$, this roughness is not believed to affect the polymer reflow during thermal annealing.
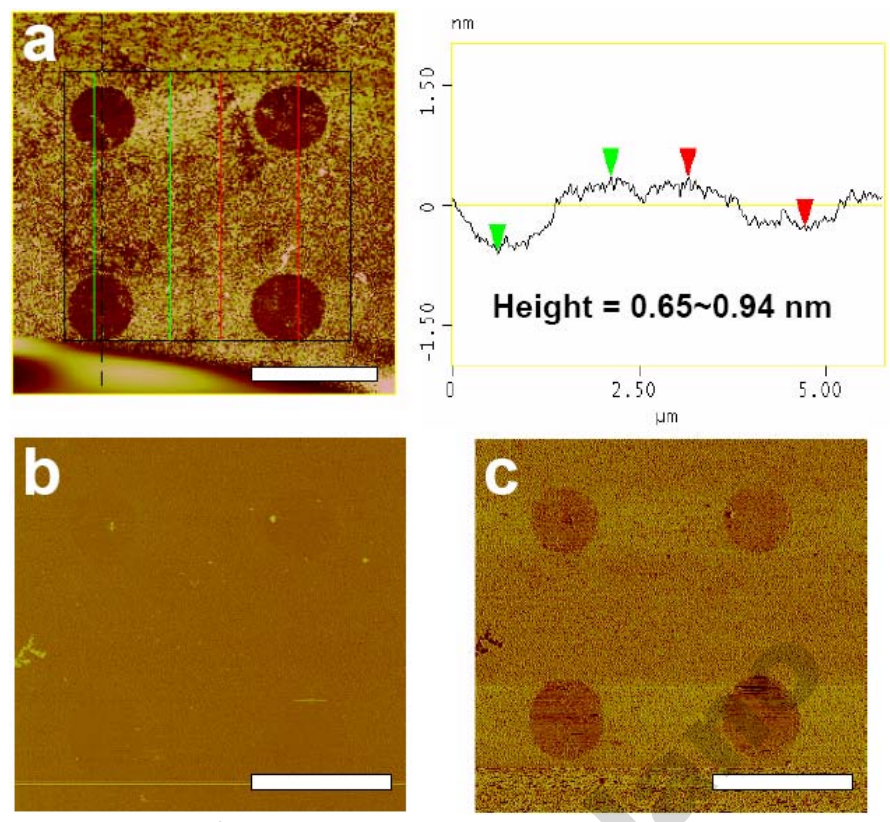

Fig. 2. AFM images of template with heterogeneous surface energies. (a) AFM topography image of FDTS treated template and cross-sectional analysis. (b) AFM topography image and (c) phase image of final template. Scale bar is $4 \mu \mathrm{m}$.

To quantify the surface energies of the templates, contact angles of deionized water and acetone on the un-patterned Si treated with only FDTS or MOPTS were measured. Their surface energies were then calculated using a two-liquid method. The surface energy of FDTS and MOPTS treated Si are $17.8 \mathrm{~mJ} / \mathrm{m}^{2}$ and $50.0 \mathrm{~mJ} / \mathrm{m}^{2}$.

\section{SEIP patterning of PEG-b-PLA and SU8}

Diblock copolymer of maleimide-terminated poly(ethylene glycol)-b-poly (D,L-lactide) (MAL-PEG-b-PLA) was used in SEIP. MAL-PEG-PLA was synthesized by ring opening polymerization of D,L-lactide at $110{ }^{\circ} \mathrm{C}$. Poly(ethylene glycol) monoethyl ether maleimide (HO-PEG-MAL, $\mathrm{M}_{\mathrm{n}}=3,210 \mathrm{Da}$ ) was used as a macro-initiator. D,L-lactide was added as a monomer and Stannous (II) octoate $\left(\mathrm{Sn}(\mathrm{Oct})_{2}\right)$ was added as a catalyst. After reacting for $4 \mathrm{~h}$, the mixture was allowed to cool down to room temperature. MAL-PEG-PLA was purified by redissolving in THF and precipitating in hexane 3 times. The degree of polymerization of the PLA was calculated by comparing integral intensity of characteristic resonance of the PLA at $5.2 \mathrm{ppm}\left(-\mathrm{C}(=\mathrm{O})-\mathrm{CH}\left(-\mathrm{CH}_{3}-\right)\right)$ and PEG resonance at $3.64 \mathrm{ppm}$ (-OCH $\left.\mathbf{H}_{2} \mathrm{CH}_{2^{-}}\right)$in the ${ }^{1} \mathrm{H}$ NMR spectrum (Figure S1). The amount of maleimide proton was calculated by comparing integral intensity of characteristic resonance at $6.70 \mathrm{ppm}$ and PEG resonance at $3.64 \mathrm{ppm}\left(-\mathrm{OCH}_{\mathbf{2}} \mathrm{CH}_{2^{-}}\right)$. The molecular weight and polydispersity of MAL-PEG-PLA were also characterized by gel permeation chromatography (THF as eluent) and the results were found to be consistent with ${ }^{1} \mathrm{H}$ NMR data. MAL-PEG-PLA $\left(\mathrm{M}_{\mathrm{n}}=7.2 \mathrm{kD}\right)$ were synthesized 
with 95\% yield and used in this study. It is noteworthy that the commercially available MAL-PEG-OH polymer has $76 \%$ of all PEG chains terminated with maleimide groups. This percentage is retained in the MAL-PEG-PLA copolymer, as demonstrated by ${ }^{1} \mathrm{H}$ NMR. This class of amphiphilic block copolymers has shown great potential as a multifunctional nanomedicine platform for loading drugs and superparamagnetic iron oxide nanoparticles (SPIO). By further attaching cell-specific ligands on the surface of the copolymer, targeted cancer detection and therapy can be achieved. Recently, co-author Gao et al. has demonstrated the self assembly of cyclic Arg-Gly-Asp peptides (cRGD) -encoded, SPIO-loaded micelles as ultra-sensitive MRI T2 agents and drug delivery systems [8], as shown in Fig 3.
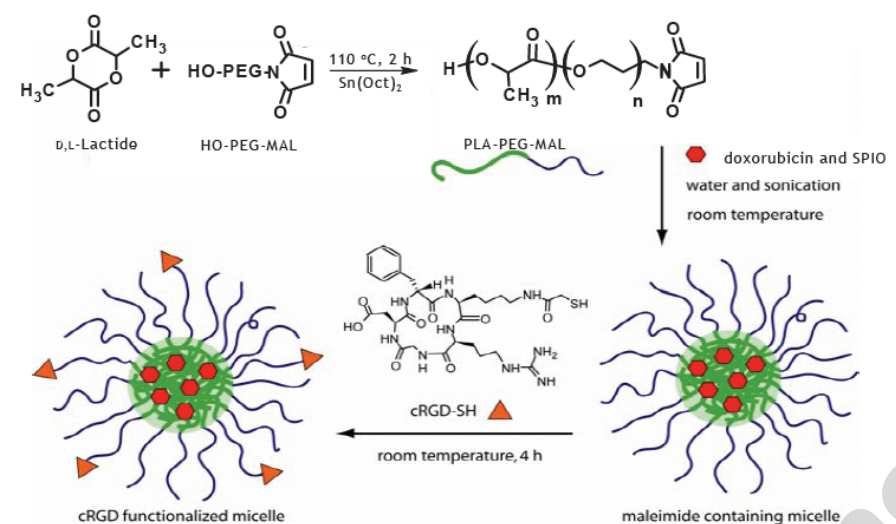

Fig. 3. Schematic diagram of synthesis of MAL-PEG-PLA copolymer and production of cRGD-functionalized, SPIO-loaded micelles.

We intend to use the SEIP process to produce PEG-b-PLA nanostructures to maximize the SPIO and/or drug loading capacity. SEIP will help better control the particle morphology and size distribution. For this purpose, PEG-b-PLA is dissolved in THF or toluene (1 1.5\% by weight ) and then spincoated onto the template. The film thickness ranges from $100 \mathrm{~nm}$ to $250 \mathrm{~nm}$ depending on polymer concentration and spincoating conditions, as indicated by profiler measurements. SU8, a UV curable epoxy-based photoresist which is a popular material for microelectronic-mechanic systems (MEMS), was also used in the SEIP process. Since SU8 has well controlled spincoat uniformity and is robust after UV radiation for consequent imaging in AFM and SEM, it is used to study and optimize the SEIP process. The polymer coated template was heated to 100 ${ }^{\circ} \mathrm{C}$ on the hot plate or in a nanoimprinter for about 10 mins. For some experiments, a gas pressure of $0.8 \mathrm{MPa}$ was applied above the film during the thermal annealing, which was found to improve the microfluidic flow of the polymer film. Figure 4 shows the patterning of PEG-PLA copolymers into residue-free discrete micro-structures. Circles $2 \mu \mathrm{m}$ in diameter and lines with $500 \mathrm{~nm}$ in width and $500 \mathrm{~nm}$ in spacing are well formed.

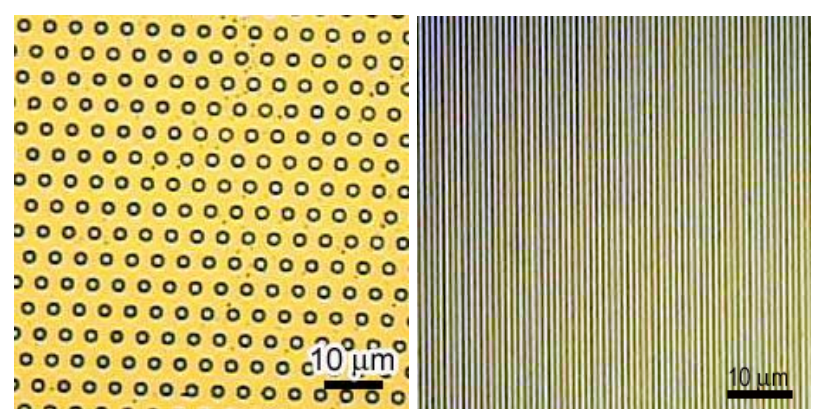

Fig. 4. Optical images of $2 \mu \mathrm{m}$ PEG-b-PLA dishes (left) and $500 \mathrm{~nm}$ wide PEG-b-PLA gratings (right) formed by heating polymer to re-flow to patterned hydrophilic areas.

These periodic polymer patterns were then imaged using AFM and results are shown in Fig. 5. Since PEG-b-PLA polymer easily sticks to the AFM tip, imaging caused material pick-up and re-deposition on the other areas, leading to the formation of shallow bands along the scanning direction. Fig.4a shows a 5 X 5 dot array with spherical morphology. The dots are about $690 \mathrm{~nm}$ in height and $2 \mu \mathrm{m}$ in diameter, which faithfully matches the underlying chemical patterns. This indicates that the polymer dimensions and shape are precisely defined by the chemical patterns on the template. Some variation in dot height was observed and one possible reason is that the initial thickness of PEG-b-PLA was relatively thick and spincoating was not uniform, which will be discussed in section III. In comparison, SU8 dots show uniform and excellent spherical morphology (Fig. 5b). The width of the particles is the same as PEG-b-PLA and the height is about $380 \mathrm{~nm}$.
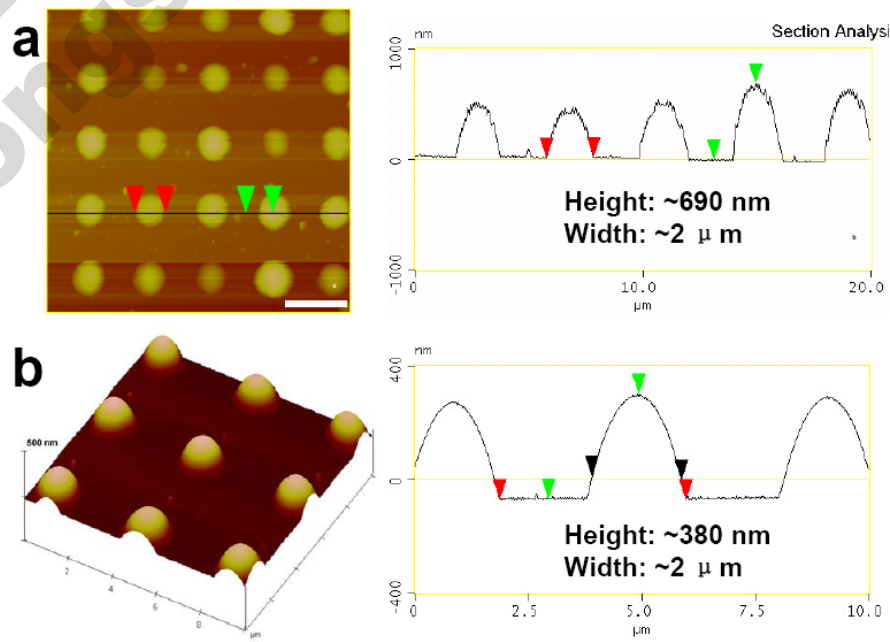

Fig. 5. AFM images of SEIP patterned polymer particles. (a) 5X5 PEG-b-PLA dot arrays and its cross-sectional analysis. (b) 3X3 SU8 dot arrays and cross-sectional analysis.

\section{Harvesting polymer particles}

The periodically patterned polymer micro and nanostructures on the solid surfaces by the SEIP can be used directly for applications in cell adhesion and growth guidance towards 
tissue engineering or in microfluidic devices. However for applications in nanomedicine, these structures must be dispersed in aqueous solution. Using a knife edge to collect patterned particles or using polydimethylsiloxane (PDMS) pads to pickup and transfer devices have been reported previously [17][18]. Both methods were tried in this study but were not feasible for the polymers we used. The polymer particles stuck to the knife edge and merged when they were scratched together. On the other hand, PDMS pads were able to pick the particles up, but the particles became stuck on the PDMS and could not be released into an aqueous solution. Instead, a probe-sonicator was used directly to lift these particles off of the Si template into an aqueous solution. As shown in Fig. 6a, the template with patterned polymer was completely submerged in a solution of $50 \%$ ethanol in deionized water (DIW). Ethanol in the solution was used to effectively wet the heterogeneous surface of the Si template. An ultrasonic probe tip, partially immersed in the solution, was used to agitate the solution with the template at $11 \mathrm{~V}$ for approximately 3 minutes while heating the solution to about $70^{\circ} \mathrm{C}$. The ultrasonic agitation in ethanol solution weakens the interfacial adhesion between the polymer and the MOPTS surface which causes the polymer particles to release from the template when scraped with a blade. After the Si template was taken out of the solution, a carbon film supported by a metal grid was immersed in the solution followed by centrifugation. In this way, the particles were collected on the carbon film and then imaged using SEM, as shown in Figure 6b. Few particles can be seen on the Si template after the harvesting and a large amount of polymer particles were seen on the carbon film. SU8 particles with diameter of 1.6-2 $\mu \mathrm{m}$ have been collected. Some of the particles can be seen on their flat sides, which results from the flat interface between the particle and the template. Currently, we are working on the harvesting of PEG-b-PLA. Since it is softer and stickier than SU8, the probe sonicator approach is more difficult. Moreover for the PEG-b-PLA polymer, we are actually interested in harvesting them into the aqueous solution via template-directed self-assembly to form micellar nanostructures in the future.
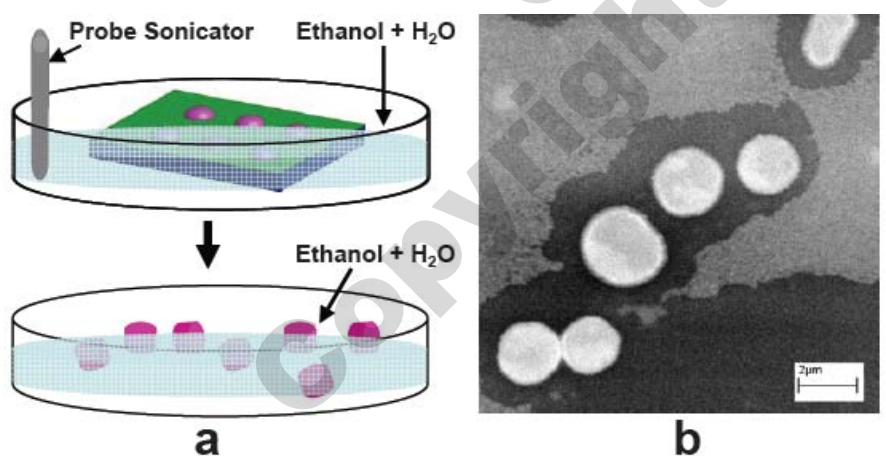

Fig. 6. Harvesting of polymer particles patterned on SEIP substrate: (a) Schematic of probe sonicator method. (b) Freestanding SU8 particles collected on a carbon membrane from DIW solution.

\section{DISCUSSION}

The underlying science of SEIP relates to polymer dynamics on heterogeneous chemical surfaces. Ultrathin films of polymer liquid (typically less than $20 \mathrm{~nm}$ ) have been intensively studied for their instability on similar surfaces[19-22]. The Van der Waals force in the polymer film and between the polymer film and engineered template surfaces are believed to drive the microfluidic behavior of polymer to re-organize onto the more wettable areas. However, since the film thickness in SEIP is much thicker than the film in previous studies, the pitch of template chemical patterns is much smaller than the spinodal wavelength [20-22]. We believe the polymer microfluidic flow is not driven by the spinodal dewetting phenomena [20-22], but by the strong surface potential gradient at the boundaries of FDTS and MOPTS treated Si surfaces in a similar way described in ref [19]. The measured height and width in Fig. 5 indicates that the contact angles of PEG-b-PLA and SU8 on the MOPTS-Si surface is about $73^{\circ}$ and $60^{\circ}$, which are much higher than their native contact angle on a large homogenous MOPTS surface. Therefore the large contact angles of SEIP particles are not governed purely by the Young's equation but supported by the FDTS-MOPTS boundary constrains around the particles. More theoretical work and simulation will be carried out in the future to quantify the effects of interface tensions.

The uniformity of initial polymer was found to be a critical factor to determine the yield and uniformity of SEIP fabrication of structures. The spincoating conditions directly affect the film uniformity, which is strongly related to the polar part of surface energy of the trichloro-silane treated Si. For example, FDTS-Si has a total surface energy of $17.8 \mathrm{~mJ} / \mathrm{m}^{2}$, where the polar part is nearly zero $\left(0.01 \mathrm{~mJ} / \mathrm{m}^{2}\right)$. This causes weak adhesion with polymer with high polarity, for example SU8. Actually SU8 cannot be directly spincoated onto the template. For SU8, we used a transfer method to deposit the film onto the template. SU8 was first spincoated onto an oxidized PDMS pad. After the restoration of its low surface energy, the polymer coated PDMS pad was pressed onto the template and slowly removed, releasing the thin polymer film [18]. For PEG-b-PLA, direct spincoating on the template is successful because the polymer has a significant amount of non-polar components that can generate adhesion with the dispersion part of FDTS-Si surface energy.

\section{SUMMARY}

We have developed a new processing technique utilizing surface energy induced polymer flow to make monodispersed and shape specific polymer structures periodically on solid surfaces. Spherical particles and cylinder lines in micro and nanoscale are formed in diblock copolymers and in UV curable epoxy polymer SU8. A probe-sonicator approach was developed to successfully liftoff structures from a solid surface 
into an aqueous solution. The SEIP technique has demonstrated much better pattern uniformity than conventional self-assembly methods and offers excellent flexibility to design particle geometry and dimensions. With these advantages and further work on encapsulation of drug and imaging agents, SEIP is believed to be a promising approach for manufacturing biomaterials for nanomedicine.

\section{REFERENCES}

[1] E. Mathiowitz et al., "Biologically erodablemicrospheres as potential oral drug delivery systems”, Nature 386, pp. 410-414, 1997.

[2] F. Qian, A. Szymanski, and J. M. Gao, "Fabrication and characterization of controlled release poly(D,L-lactide-co-glycolide) millirods”, J. Biomed. Mater. Res. 55 (4), pp. 512-522, 2001.

[3] V. P. Torchilin, "Targeted polymeric micelles for delivery of poorly soluble drugs", Cell Mol Life Sci. 61(19-20), pp. 2549-2559, 2004.

[4] F. Ahmed and D. E. Discher, "Self-porating polymersomes of PEGPLA and PEG-PCL: hydrolysis-triggered controlled release vesicles", $J$ Control Release 96, pp.37-53, 2004.

[5] N. A. Peppas, "Intelligent biomaterials as pharmaceutical carriers in microfabricated and nanoscale devices”, MRS Bulletin 31 (11), pp. 888-893, 2006.

[6] G. B. Sukhorukov and H. Mohwald, "Multifunctional cargo systems for biotechnology”, Trends Biotechnol. 25 (3), pp. 93-98, 2007.

[7] B. Sitharaman, K. R. Kissell, K. B. Hartman, L. A. Tran, A. Baikalov, I. Rusakova, Y. Sun, H. A. Khant, S. J. Ludtke, W. Chiu, S. Laus, E. Toth, L. Helm, A. E. Merbach, and L. J. Wilson, "Superparamagnetic gadonanotubes are high-performance MRI contrast agents", Chem. Commun. 31, pp. 3915-3917, 2005.

[8] N. Nasongkla, E. Bey, J. Ren, H. Ai, C. Khemtong, J. Setti Guthi, S. Chin, A. D. Sherry, D. Boothman, and J. Gao, "Multifunctional Polymeric Micelles as Cancer-Targeted, MRI-Ultrasensitive Drug Delivery Systems," Nano Lett., (6)11, pp. 2427-2430, 2006.

[9] A. Curtis and C. Wilkinson, "Nantotechniques and approaches in biotechnology”, Trends Biotechnol. 19 (3), pp.97-101, 2001.

[10] W. Hu , E. Yim , R. M. Reano , K. W. Leong, and S. W. Pang, " Effects of nanoimprinted patterns in tissue-culture polystyrene on cell behavior", J. Vac. Sci. Technol. B. 23(6), pp. 2984-2989, 2005.

[11] A. S. Andersson, F. Backhed, A. von Euler, et al., "Nanoscale features influence epithelial cell morphology and cytokine production", Biomater. 24 (20), pp. 3427-3436, 2003.

[12] S. Y. Chou, P. R. Krauss, and P. J. Renstrom, "Nanoimprint lithography", J. Vac. Sci. Technol. B. 14 (6), pp. 4129-4133, 1996.

[13] Y. Xia and G. M. Whitesides, "Soft lithography", Annu. Rev. Mater. Sci. 28, pp. 153-84, 1998

[14] O. Cherniavskaya, A. Adzic, C. Knutson, B. J. Gross, L. Zang, et al., "Edge transfer lithography of molecular and nanoparticle materials", Langmuir 18, pp. 7029-34, 2002.

[15] T. W. Odom, V. R. Thalladi, J. C. Love, and G. M. Whitesides, “ Generation of 30-50 nm structures using easily fabricated, composite PDMS masks", J. Am. Chem. Soc. 124, pp. 12112-13, 2002

[16] S. Y. Chou and L. Zhuang, "Lithographically induced self-assembly of periodic polymer micropillar arrays”, J. Vac. Sci. Technol. B. 17 (6), pp. 3197-3202, 1999.

[17] J. P. Rolland, B. W. Maynor, L. E. Euliss, A. E. Exner, G. M. Denison, and J. M. DeSimone, "Direct Fabrication and Harvesting of Monodisperse, Shape-Specific Nanobiomaterials,” J. Am. Chem. Soc 127 (28), pp. 10096 $-10100,2005$.

[18] M. A. Meitl, Z. T. Zhu, V. Kumar, K. J. Lee, X. Feng, Y. Y. Huang, I. Adesida, R. G. Nuzzo, J. A. Rogers, "Transfer printing by kinetic control of adhesion to an elastomeric stamp", Nature Mater. 5 (1), pp. 33-38, 2006.

[19] H. Gau, S. Herminghaus, P. Lenz, and R. Lipowsky, "Liquid morphologies on structured surfaces: from microchannels to microchips", Science 283, pp. 46-49, 1999.
[20] A. M. Higgins and R. A. L. Jones, "Anisotropic spinodal dewetting as a route to self-assembly of patterned surfaces”, Nature 404 (6777), pp. 476-478, 2000.

[21] A. Sehgal, V. Ferreiro, J. F. Douglas, E. J. Amis, and A. Karim, “Pattern-directed dewetting of ultrathin polymer films", Langmuir 18(18), pp. 7041-7048, 2002.

[22] K. Kargupta and A. Sharma, "Templating of thin films induced by dewetting on patterned surfaces”, Phys. Rev. Lett. 86, pp. 4536, 2001.

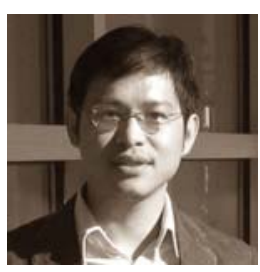

Dr. Wenchuang (Walter) Hu (M’04) received the B.S. degree from Peking University, Beijing, China, in 1999, and the M.S.E.E. and Ph.D. degrees from the University of Notre Dame, Notre Dame, IN, in 2001 and 2004, respectively. Then, he spent a year as a Post-Doctoral Research Fellow at the Solid State Electronics Laboratory, Department of Electrical Engineering and Computer Science, University of Michigan, Ann Arbor, MI. In September 2005, he joined the Department of Electrical Engineering of the University of Texas at Dallas as an assistant professor. His research interests include engineering nanostructured biomaterials, metrology for polymer nanostructures, and fabrication of electrical and optical nano-devices using a combination of nanolithography and self assembly. His expertise is in the areas of electron beam lithography, nanoimprint lithography, and nanofabrication processes and metrology. He is familiar with surface chemistry, polymer science, and cell biology. He is an active member of Sigma Xi, IEEE, MRS, SPIE, and AVS.

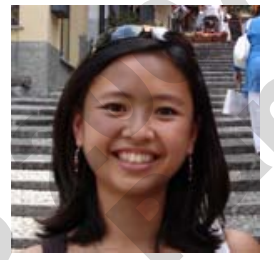

Fern Yoon received her B.S. degree in Electrical Engineering from the University of Texas at Dallas in 2007. She will continue on as a Get-Doc recipient at the University of Texas at Dallas pursuing a M.S. /Ph.D. degree also in Electrical Engineering. In the spring of 2007, she spent the whole semester at the University of Texas Southwestern Medical School at the Simmons Comprehensive Cancer Center as a Green Fellow, doing research to combine top-down engineering with bottom-up chemistry approach for enhanced cancer drug delivery. She is an active member of SWE and has served as President and Vice President of the SWE section at UT Dallas.

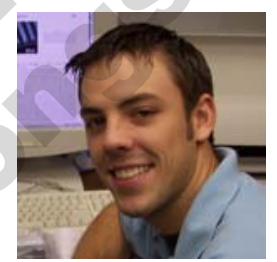

Adam Crouch (M'05) received his B.S. degree in Engineering Science from Trinity University in San Antonio, TX in 2002. He is currently pursuing a M.S.E.E. and Ph.D. degree from the University of Texas at Dallas in Microelectronics in the Department of Electrical Engineering. His research interests include fabricating nanostructured and complex 3-D biomaterials for cellular studies, surface energy induced patterning of polymers, and emerging nanoimprint technologies. He is an active member of IEEE and MRS.

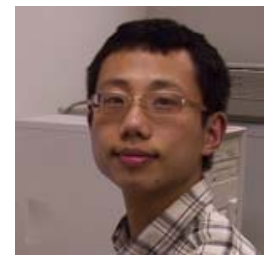

Li Tao (M’06) received his B.S. degree (with honors) in Materials Science and Engineering from Southeast University, Nanjing, China, in 2004 and then worked as a research assistant on a Chinese national 863 funded project. Since 2006, he has been a graduate student and research assistant in the Department of Electrical Engineering of the University of Texas at Dallas, Richardson, TX. His research interests include electron beam lithography, nanoimprint lithography, nano-characterization, and biomedical nano-devices. He is a student member of IEEE and Material Research Society (MRS) since 2006.

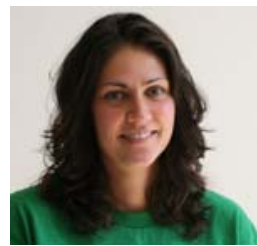

Dr. Heather Hillebrenner received her B.A. degree in both chemistry and biology from Southern Illinois University Edwardsville in Edwardsville, Illinois, in 2002 and her Ph.D. in chemistry from the University of Florida in Gainesville, Florida in 2006. She is currently a Post-Doctoral Research Associate and Komen Post-Doctoral Fellow at the Simmons 
Comprehensive Cancer Center at the University of Texas Southwestern Medical Center in Dallas, Texas. Her research interests include the development of novel magnetic resonance imaging contrast agent using the combination of synthetic chemistry and materials science. Her expertise lies in the area of synthesis.

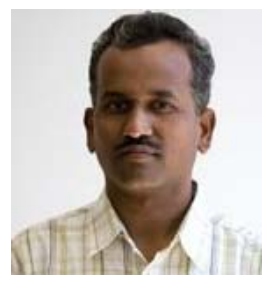

Dr. Jagadeesh Setti Guthi received his M.Sc and Ph.D degrees in Organic Chemistry from the Department of Chemistry, Osmania University, Hyderabad, India, in 1993, and 1999, respectively. He then spent a year as a Research Associate in the Department of Organic Chemistry, Indian Institute of Science, Bangalore, India. In May 2002, he conducted his Post-Doctoral Research training in the Department of Biochemistry, at the University of

Texas Southwestern Medical Center at Dallas. In January 2006 he joined the Simmons Comprehensive Cancer Center, University of Texas Southwestern Medical Center at Dallas, as a Research Associate. His expertise involves total synthesis of natural products, drugs, peptides. His research interests include synthesis of novel amphiphilic block copolymers, peptides and drugs for cancer-targeted imaging and drug delivery applications.

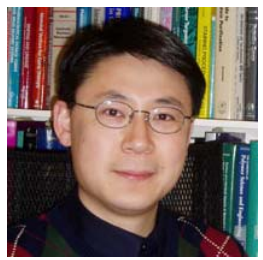

Dr. Jinming Gao received his B.S. degree in Chemistry at Peking University (Beijing, China) in June 1991. In May 1996, he received a Ph.D. degree in Medicinal Chemistry from Harvard University. Following his doctoral studies, he completed his postdoctoral training in Biomedical Engineering at Massachusetts Institute of Technology. In August 1998, Dr. Gao joined the faculty of Biomedica Engineering at Case Western Reserve University in Cleveland, Ohio. He was promoted to Associate Professor (with tenure) of Biomedical Engineering and Radiology in April 2004. In July 2005, Dr. Gao moved his lab to Dallas, Texas, where he holds a primary appointment as Associate Professor (with tenure) of Oncology and Pharmacology in the Simmons Comprehensive Cancer Center at UT Southwestern Medical Center and a joint appointment as Associate Professor of Chemistry at UT Dallas. He is also the Founding Member of the Bioengineering Department at UT Dallas. Dr.Gao's expertise is in biopolymers, macromolecular self assembly, nanocomposite materials, MR contrast agents, and cancer nanomedicine. Dr. Gao has published over 50 peer reviewed papers, 40 conference proceedings and three book chapters. He is currently serving as the Associate Editor for Experimental Biology and Medicine and is also a visiting professor at Zhongshan University in Guang Zhou, China. Using a highly-interdisciplinary approach, his lab is focused on development of multi-functional nanomedicine platforms that incorporate tumor targeting, imaging ultrasensitivity, and controlled drug release for cancer-targeted therapy. 